\title{
L'Ospite inatteso
}

\section{Massimo Livi Bacci}

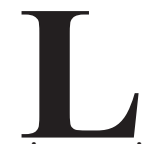

a storia è fin troppo nota. Nel Dicembre del 2019, le autorità sanitarie di Wuhan, capitale di 11 milioni di abitanti della provincia dello Hubei, riscontrarono un numero abnorme di pazienti con sintomi di polmonite provocata da "cause sconosciute", ma subito collegate a una zoonosi avvenuta nel mercato "umido" della città, dove si vendono pesce e animali vivi e nel quale lavoravano molti degli ammalati. Il 12 dicembre l'esistenza del focolaio infettivo viene annunciato alla televisione di stato, il 31 viene avvertita l'Organizzazione Mondiale della Sanità, il 7 gennaio le autorità cinesi dichiarano di avere individuato il nuovo virus (SARS-CoV-2, causa della malattia poi ufficialmente designata Covid-19'1); il 22 Gennaio viene dichiarata la rigorosa quarantena a Wuhan, poi estesa a tutta la provincia (60 milioni di abitanti). L'epidemia si diffonde rapidamente in altri paesi del sud-est asiatico e poi in Europa e negli altri continenti. L'11 marzo l'OMS, fino ad allora molto cauta nella valutazione della pericolosità dell'epidemia, si decide a promuovere il Covid-19 al grado di "pandemia" (una definizione puramente burocratico-tassonomica ma con implicazioni politiche rilevanti). Il direttore Ghebreyesus in una conferenza stampa proclama lo stato pandemico, visto che "nelle ultime tre settimane il numero dei casi di Covid-19 al di fuori della Cina è aumentato di 13 volte e il numero di paesi colpiti è triplicato, e che ci sono più di 118,000 casi in 114 paesi e 4,291 persone hanno perso la vita ${ }^{2}$ ". Meglio tardi che mai! Anche i ciechi si erano accorti da tempo che il contagio si sarebbe diffuso in tutto il mondo; gran parte dei paesi avevano limitato spostamenti e voli, operato serrate (lockdown) locali (Lodi, Codogno e Vo' in Italia); proclamato quarantene o la serrata generale (il 9 marzo, in Italia).

\section{UN'ARMA ANTICA SEMPRE EFFICACE: L'ISOLAMENTO}

Come dai tempi della peste, la carta vincente contro la diffusione del contagio è ancor oggi l'isolamento degli infetti, il rallentamento, quando non

\footnotetext{
1 Acronimo di COronaVIrus Disease, col 19 che indica l'anno di insorgenza.

2 Sicuramente una dichiarazione tardiva! http:/www.salute.gov.it/portale/nuovocoronavirus/dettaglioNotizieNuovoCoronavirus.jsp?lingua $=$ italiano $\&$ menu $=$ notizie $\& p=$ dalministero $\& i d=4209$
} 
il blocco totale, della mobilità e dei contatti interpersonali. ${ }^{3}$ In assenza di vaccini e di farmaci specifici risolutivi, la ricetta secolare è stata adottata quasi ovunque, con maggiore o minore rigore e con varia tempistica. Alcuni paesi — come il Regno Unito e gli Stati Uniti- si sono cullati nell'idea che si potesse raggiungere una immunità di gregge senza troppe perdite umane (e soprattutto economiche), salvo a ricredersi e imboccare precipitosamente la via tradizionale. Altri paesi — come la Svezia - hanno percorso una via intermedia, contando sull'ottimo sistema sanitario, il senso civico e di autocontrollo della popolazione. Ma non ha funzionato troppo bene, come conferma il confronto con gli altri paesi scandinavi e il fatto che le vittime del coronavirus sono al momento nel quale scriviamo allo stesso livello dell'Italia, il paese Europeo che per primo è stato toccato dal virus e che per primo ha messo in atto un lockdown generalizzato, e superiori a quelle della Francia. ${ }^{4} \mathrm{Nel}$ cuore dell'Europa, la Germania, che ha avuto perdite relative tra un quarto e un sesto rispetto agli altri grandi paesi europei, è un caso eccezionale del quale vanno indagate le ragioni. Forse il fatto che il sistema sanitario abbia preso rapidamente in carico le persone sintomatiche, curandole per tempo, ha permesso di fare sopravvivere persone che altrimenti non ce l'avrebbero fatta. Ma potrebbero anche esserci particolarità nei metodi di individuazione della causa del decesso e delle sue concause, che hanno determinato un conteggio dei deceduti per causa del coronavirus inferiore al (presunto) vero. Non sappiamo. Eppure la Germania è un paese con alta densità abitativa, forte mobilità interna e internazionale, grandi metropoli, al centro di una fittissima rete di contatti e scambi internazionali: caratteristiche ideali per essere recettore e diffusore di infezioni e contagi. Non a caso nel mondo le grandi aree metropolitane sono state, in questa epidemia, i centri propulsori (oltre che più colpiti) del contagio: Milano e la Lombardia, Madrid, Parigi, Londra, New York, Città del Messico, San Paolo. Non manca materia di studio per gli epidemiologi.

\section{L'OSPITE INATTESO}

Il coronavirus, nella sua forma attuale, è stato un Ospite inatteso, e non sappiamo ancora quando potremo liberarcene. Non si sa se, e dove, ci sarà un'ondata successiva a quella attuale, che sta attualmente rifluendo in mol-

\footnotetext{
3 Tradotto colpevolmente in "distanziamento sociale", anziché "fisico".

${ }^{4}$ Al 15 Agosto 2020, il numero di vittime per milione di abitanti, era pari - nei grandi paesi Europei - a 612 in Spagna, 609 nel Regno Unito, 583 in Italia, 572 in Svezia, 466 in Francia, 111 in Germania e 107 in Russia. In America, alla stessa data, 518 negli Usa, 501 in Brasile, 433 in Messico. Ma la diversa dinamica dei contagi nel vari paesi potrebbe far cambiare rapidamente questa graduatoria nei prossimi mesi.
} 
ti paesi. Non si sa se dovremo convivere, e quanto a lungo, con il virus, o se questo si adatterà agli umani in modo meno virulento. Non si sa se, e quando, farmaci o vaccini ci potranno curare o preservare. E poi l'Ospite inatteso ha anche comportamenti non conformi ad altri virus della stessa natura (un illustre scienziato ha argutamente detto "non ha studiato sui libri di immunologia") $)^{5}$ : ci sono dubbi sulla durata e infettività del periodo di latenza, sul perché punisca più gli uomini che le donne, se e quanto a lungo produca immunità in chi lo ha subito; va chiarita la sua contagiosità (tramite gli indici R di riproduttività) e la sua letalità. Certo si sta studiando attentamente il suo comportamento, e i sistemi sanitari sono, qualche mese dopo il suo arrivo, più capaci di difendere chi viene contagiato.

Che il Covid-19 abbia colpito impreparati i paesi, anche quelli più moderni, può spiegarsi storicamente. Verso la metà del secolo scorso, si pensava di essere sulla strada giusta per alleviare, o addirittura eliminare, il fardello delle patologie infettive e trasmissibili. Antibiotici, vaccini, controllo dei vettori, igiene pubblica avevano fatto rapidamente progredire la sopravvivenza. Malattie secolari erano state sradicate. La potenza di fuoco della scienza si diresse, allora, verso le patologie non trasmissibili, quelle dei sistemi cardiocircolatorio o neurologico, il cancro. L'emergere di nuove patologie infettive o il riemergere di quelle antiche, credute debellate, apparivano fenomeni circoscritti e comunque controllabili. Fino all'emergere dell'Aids, negli anni '80, che fu un tragico richiamo al fatto che il mondo biologico è sempre in movimento e che i rapporti tra microbi, vettori, animali, ambiente e umani è in continua, imprevisibile evoluzione. Ebola, SARS, MERS, Dengue, Lassa, Lyme e molte altre patologie, hanno avuto effetti prevalentemente locali e numericamente modesti. Non così l'Aids (forse 30 milioni di decessi nel mondo) che però ha avuto caratteristiche assai diverse dalle epidemie tradizionali, assai più legata ai comportamenti individuali, e concettualmente più vicina all'abuso di oppioidi, al tabagismo o all'alcolismo, che non agli impalpabili contagi delle epidemie classiche. In Italia, ma anche in molti altri paesi, i progenitori più recenti del Covid-19, le pandemie influenzali Asiatica del 1957-58, e Hong Kong del 1968-69, hanno causato solo modeste increspature sulla curva dei decessi, simili a quelle verificatesi in anni di eccessi climatici, di gran freddo o di grandi calure. Tutto questo spiega perché il mondo si sia trovato impreparato ad affrontare la nuova epidemia; la guardia era stata abbassata, la sorveglianza attenuata, le protezioni più semplici (come le mascherine)

\footnotetext{
5 Alberto Mantovani, intervistato da Cristina Marrone: "Questo virus non ha studiato sui libri di immunologia e si comporta in modo diverso da quanto siamo abituati a vedere", Corriere della Sera, 28 Maggio 2020, p. 13
} 
introvabili, le risorse della sanità più preparate alla guerra di lungo corso contro le patologie tumorali e cardiocircolatorie, che non ai blitz epidemici. Va però osservato che alcuni paesi si sono fatti trovare assai più impreparati di altri all'arrivo dell'epidemie, e l'hanno gestita malamente.

\section{Un CONFronto IMPARI: SPAgnola E Covid-19}

E' naturale che scienziati, storici e media confrontino l'epidemia Covid-19 con la Spagnola ${ }^{6}$ di un secolo fa, rilevando analogie e differenze. Analogie, per quanto riguarda contagiosità e letalità; differenze per quanto riguarda le caratteristiche delle vittime e la loro numerosità (l'unità di conto, per la Spagnola e a livello planetario, sono le decine di milioni) e, soprattutto, l'accresciuta capacità, oggi, di far fronte al virus. Un confronto appropriato potrà farsi solamente quando l'epidemia, con le sue eventuali ricadute, si sarà esaurita. Si possono però fare dei rozzi confronti su alcune "grandezze", anzi "ordini di grandezza", di natura demografica e sociale, relativi all'Italia. Per la durata possiamo dire che fu di 10 mesi per la Spagnola, tra prima e seconda ondata: impossibile è fare una previsione per l'epidemia attuale, la cui prima ondata si sta esaurendo dopo quattro mesi. In Italia, i decessi stimati per la Spagnola, da una recente e approfondita ricerca basata sui dati dello stato civile e sulle statistiche militari, furono $466,000^{7}$ (su una popolazione di 36 milioni di abitanti nel 1918). Per quella attuale - che fino al 15 Agosto ha fatto 35,000 vittime - possiamo avventurarci a ipotizzare un bilancio finale pari a 50,000, ${ }^{8}$ includendo le sottostime, e una eventuale ma attenuata nuova ondata. su una popolazione di 60 milioni; con una mortalità pari a un sedicesimo rispetto alla Spagnola. ${ }^{9}$ Ma le vittime della Spagnola furono prevalentemente giovani, con una età media minore di 30 anni, e si stima che, mediamente, perdessero 29 anni di vita: in totale quasi 14 milioni di anni di vita perduti. Secondo i rilievi dell'Istituto

\footnotetext{
${ }^{6}$ Ricordiamo che l'aggettivo Spagnola attribuito a questa forma virale, deriva da un equivoco storico. Nella forma iniziale più blanda, l'epidemia influenzale sorse — sembra - nel cuore degli Stati Uniti nell'Aprile del 1918, nei campi che ospitavano reclute destinate all'intervento in Europa; che quando l'epidemia si diffuse in Europa ne parlarono dettagliatamente i giornali in Spagna, che essendo neutrale, non aveva censura. Censura che invece esisteva in tutti gli altri paesi belligeranti, che per motivi di sicurezza, lasciavano filtrare poche notizie sulla rapidità e violenza dell'epidemia.

7 A. Fornasin, M. Breschi, M. Manfredini, Spanish Flu in Italy: New data, new questions, "Le infezioni in Medicina", (26), 1, 2018, p. 101.

${ }^{8}$ Con l'andamento attuale della curva dei decessi, il numero finale di questi, all'esaurirsi dell'epidemia, sarebbe intorno alle 35,000 unità. Per il criterio di calcolo, si veda www.Neodemos. info. Supponendo un $20 \%$ di sottostima, e un'eventuale seconda ondata di virulenza pari a un quinto della prima, si arriva a 50,000. Si tratta naturalmente di ipotesi, col solo fine di indicare un ordine di grandezza possibile.

9 La mortalità dell'Epidemia: 50,000/60 milioni $=0.08$ per mille; quella della Spagnola, $466.000 / 36$ milioni $=1.3$ per 1000 , cioè 16 volte più grande.
} 
Superiore della Sanità (ISS), l'età media dei deceduti è stata pari a 80 anni; ciascuno di questi (ancora in media) ha perduto al più 10 anni di vita, con un bilancio totale di 0.5 milioni di anni di vita non vissuti (circa la 28esima parte rispetto alla Spagnola) ${ }^{10}$. La Spagnola uccise 200 mila persone tra $\mathrm{i}$ 20 e i 50 anni, e di conseguenza centinaia di migliaia di persone rimasero vedove, o vedovi, o orfani in minore età. Le vittime dell'epidemia attuale sotto i 50 anni sono state, secondo le rilevazione dell'ISS appena 1'1 per cento del totale, e vedove e vedovi sotto i 50 anni, e i loro figli minori rimasti orfani saranno, a fine epidemia, solo qualche centinaio.

\section{DUE FALSI COLPEVOLI: SVILUPPO E GLOBALIZZAZIONE}

Molte, varie e interessanti considerazioni si stanno facendo sull'arrivo dell'Ospite inatteso; occorrerà raccoglierle e discuterle con pazienza. $\mathrm{Ne}$ scelgo una, di natura molto generale e di particolare rilevanza, secondo la quale il sorgere dell'epidemia è la conseguenza dell'intrusione umana nei delicati equilibri naturali, a riprova dell'insostenibilità delle attività produttive e consumistiche di una massa crescente di persone, oramai prossima alla soglia degli 8 miliardi. E, per di più, in un mondo sempre più mobile, interconnesso e globalizzato. L'epidemia è, dunque, il sintomo dell'insostenibilità dello sviluppo. Ci sono sicuramente alcuni elementi di verità in questa considerazione, ma l'emergenza di nuove patologie - come quella attuale - non ne è l'inevitabile conseguenza. L'intrusione degli umani negli ambienti naturali è antica quanto l'umanità, e l'interazione umani-animali e le zoonosi che ne sono sorte, sono all'origine di gran parte delle patologie trasmissibili, dall'influenza alla peste. Anzi, forse, questa interazione era, nel passato, assai più intensa: si pensi nelle nostre campagne, ai pastori con le loro greggi, alle famiglie contadine conviventi con gli animali da cortile e coabitanti con gli animali nelle stalle, ai cacciatori nei boschi e nelle paludi. Quanto alla globalizzazione, è vero che essa mette in contatto anche gli angoli più remoti della terra, fa viaggiare rapidamente microbi e virus da un gruppo umano a un altro. Le popolazioni americane non avevano mai conosciuto né il vaiolo né il morbillo fin quando gli imprudenti navigatori misero in contatto l'Eurasia e l'Africa col Nuovo Mondo, completando quella che Le Roy Ladurie aveva chiamato unification microbienne du monde. Peraltro, il resto del mondo era già unificato prima di Colombo: la peste bubbonica originaria dell'estremo oriente in

${ }^{10}$ L'età media dei deceduti di Spagnola fu di 27.9 anni; gli anni di vita perduti sono calcolati moltiplicando il numero dei deceduti in ciascuna classe per la differenza tra la speranza di vita a quell'età (secondo le tavole di mortalità del 1921-22) e l'età alla morte. Stessa procedura di calcolo per l'epidemia di Covid-19. 
pochi anni fece il giro dell'Europa, della Russia e dell'Africa settentrionali, sette secoli prima che si sentisse parlare della Via della Seta (o BRI, Belt and Road Initiative) cinese. La Spagnola, che nella sua forma più blanda prese avvio nel cuore degli Stati Uniti nella primavera del 1918 (cfr. nota 6), a fine anno si era già diffusa in quasi tutto il mondo, pur in assenza di un traffico aereo che accorciasse le distanze. Possiamo dire perciò che le condizioni per la veloce diffusione di un virus esistevano già da tempo, assai prima che la mobilità delle merci e delle persone prendesse il ritmo frenetico degli ultimi decenni.

\section{E ADESSO?}

Alla soglia dell'autunno del 2020, l'epidemia, in alcune parti del mondo, è ancora in fase di ascesa, in altre ha esaurito la sua spinta, ma continua a serpeggiare e a riemergere in una pluralità di focolai. La notevole potenza di fuoco della scienza è diretta a distruggere il virus con farmaci efficienti, oppure a prevenirne l'insorgere con i vaccini. Ma ci vorrà del tempo, ci vorranno grandi sforzi per la sperimentazione, per la produzione e per la diffusione in quantità miliardarie dei ritrovati più efficienti. Ci vorrà impegno sociale e sanitario per individuare sul nascere i nuovi focolai e per spengerli o circoscriverli. Ci vorrà impegno politico per incanalare i cittadini in comportamenti prudenti. Ci vorrà impegno finanziario per reperire le risorse necessarie. Etica e civismo dovranno guidare i governi nelle loro decisioni: per contemperare obblighi e divieti con alcuni fondamentali diritti individuali dati per scontati; per individuare le priorità di impiego di risorse limitate; per costruire piani condivisi per la distribuzione di vaccini e farmaci se disponibili in quantità non sufficienti per tutti.

L'Ospite inatteso, intanto, resta con noi. Più che in una sua partenza, contiamo, prima di dargli uno sfratto vaccinale, in un reciproco e pacifico adattamento.

\section{SOMMARIO CURRICULUM DELL'AUTORE}

\section{Massimo Livi Bacci}

Es profesor de Demografía en la Universidad de Florencia y ha formado demógrafos en varios países americanos entre los que destacan Estados Unidos, México, Brasil y también en algunos países europeos. Su labor científica se ha centrado en diversos aspectos de la Demografía histórica y la Demografía actual. Es partidario de un enfoque interdisciplinario de los estudios de población y considera que la expansión a otros campos, 
más allá de la Demografía, pero desde ella, es esencial para obtener una explicación correcta de los grandes temas. Mantiene dos líneas de investigación: Poblaciones nativas de Iberoamérica después de la conquista, investigación en el ámbito de la Demografía histórica e Interacción entre los cambios demográficos y las políticas sociales. Ha publicado numerosos libros, ensayos y artículos y ha dirigido grupos de investigación. Es cofundador de sociedades científicas, en colaboración con instituciones públicas y privadas, tanto en Italia como en el resto del mundo.

Dirección electrónica: 1ivi@disia.unifi.it 